\title{
Adaptación al español del Inventario Multidimensional de Fatiga al entorno laboral*
}

\section{Adaptation into Spanish of the Multidimensional Fatigue Inventory in Work Environment}

Recibido: enero 13 de 2014 | Revisado: enero 13 de 2014 | Aceptado: abril 9 de 2014

\author{
JOAN BOADA-GRAU ** \\ Universitat Rovira i Virgili, Tarragona, España \\ ENRIQUE MERINO-TEJEDOR *** \\ Universidad de Valladolid, España \\ CARME GIL-RIPOLL ***** \\ Escuela de Alta Dirección y Administración, \\ Barcelona, España \\ GEMMA SEgARRA-PÉREZ ****** \\ Departamento de Salud de la Generalitat de \\ Catalunya, España \\ ANDREU VIGIL-COLET $* * * * * *$ \\ Universitat Rovira i Virgili, Tarragona, España
}

doi.org/10.11144/Javeriana.UPSY13-4.aeim

Para citar este artículo: Boada-Grau, J., MerinoTejedor, E., Gil-Ripoll, C., Segarra-Pérez, G., \& Vigil-Colet, A. (2014). Adaptación al español del Inventario Multidimensional de Fatiga (MFI) al entorno laboral. Universitas Psychologica, 13(4), 1279-1287. http://dx.doi.org/10.11144/Javeriana. UPSY13-4.aeim

* Artículo de investigación

** PhD. Profesor Titular de Universidad. Centro de Investigación de Evaluación y Medida de la Conducta (CRAMC). Correo electrónico: joan. boada@urv.cat

**** Correo electrónico: enmerino@psi.uva.es

***** Correo electrónico: cgil@eada.edu

******* Agencia de Salud Pública. Correo electrónico: usl.tarragona@gencat.cat

******* PhD. Catedrático de Universidad. Centro de Investigación de Evaluación y Medida de la Conducta (CRAMC). Correo electrónico: andreu. vigil@urv.cat

\section{RESUMEN}

La necesidad de evaluar la fatiga al entorno laboral es esencial para poder desarrollar programas de prevención e intervención. El principal objetivo de este trabajo fue adaptar del inglés al español el Inventario Multidimensional de Fatiga. Se analizaron la estructura interna y la fiabilidad, y se aportaron evidencias de validez convergente. La aplicación de la escala se realizó a una muestra multiocupacional compuesta por 414 empleados. También se utilizaron varios correlatos externos y dos escalas de contraste. Se aplicó el análisis factorial tanto exploratorio como confirmatorio. Los resultados indican que la versión española está conformada por 19 ítems y tres factores denominados Fatiga General, Fatiga Física y Concentración/ Motivación. Los coeficientes de fiabilidad obtenidos fueron adecuados y, además, se obtuvieron evidencias de validez con cuatro correlatos externos y dos escalas de fatiga. La presente escala adaptada al español muestra unas propiedades psicométricas idóneas y puede resultar adecuada para identificar de manera apropiada la fatiga en entornos laborales. Futuras investigaciones podrían utilizarla como una herramienta de screening en combinación con otros instrumentos.

Palabras clave

Salud laboral; vigilancia de la salud; fatiga laboral; fatiga percibida; versión española; instrumento psicométrico

\section{A B S T R A C T}

Evaluation of fatigue in work environment is crucial in order to draw up prevention and intervention programmes. The main objective of this study is to adapt the Multidimensional Fatigue Inventory from English into Spanish. I analysed the internal structure and reliability and evidence was found of convergent validity. The scale was applied to a multi-occupational sample made up of 414 employees. Various external correlates and two contrast scales were also used. An exploratory as well as a confirmatory factor analysis were undertaken. The findings indicate that the Spanish version which we have presented is made up of nineteen items and three factors, namely Gen- 
eral Fatigue, Physical Fatigue and Concentration/Motivation. The resulting reliability coefficients were adequate and, what's more, evidence of validity was found for four external correlates and two fatigue scales. The present scale, adapted into Spanish displays ideal psychometric properties and may prove ideal for adequately identifying fatigue in work environments. The scale could be used in future research as a screening tool in combination with other instruments. Keywords

Occupational health; health surveillance; occupational fatigue; perceived fatigue; Spanish version; psychometric instrument

La fatiga se ha reseñado como una amenaza a la calidad de vida cuando sobrepasa unos umbrales excesivos y, además, cabe destacar que es un problema habitual tanto en entornos clínicos como laborales de los países industrializados (González, Moreno, Garrosa, \& López 2005). La fatiga es común en la población general (Dittner, Wessely, \& Brown, 2004), al ser el resultado de un desequilibrio entre las exigencias y los recursos de la persona que da lugar a una serie de consecuencias entre las que se encuentra la fatiga. Es conocido que la fatiga disminuye la eficiencia en el trabajo y afecta negativamente tanto al rendimiento de los trabajadores como a su salud y, más allá, afecta a la sostenibilidad de las organizaciones (Herusasongko, Sutomo, \& Sudibyakto, 2012).

La fatiga se define como cansancio extremo y persistente, debilidad o agotamiento mental o físico (o ambos) (Dittner et al., 2004). También se conceptualiza como el cambio en el mecanismo del control psicológico que regula el comportamiento de una tarea, que es el resultado de los estados mentales preliminares y/o de los esfuerzos físicos, todo ello se convierte en una molestia para la persona hasta tal punto que esta ya no es capaz de responder adecuadamente a las exigencias mentales que su trabajo requiere, así el individuo es capaz de satisfacer estas demandas a costa de aumentar el esfuerzo mental y la superación de sus resistencias psíquicas (Beurskens, Bültmann, Kant, Vercoulen, Bleijenberg, \& Swaen, 2000).

La fatiga laboral está inducida por las características individuales de los trabajadores como el ritmo circadiano, la edad, el sueño y la nutrición (Satterfield \& Van Dongen, 2013) y, también, por los aspectos laborales como la carga de trabajo, la duración del trabajo y la turnicidad (Roach, Fletcher, \& Dawson, 2004). Las consecuencias de la fatiga se manifiestan por un decrecimiento de la motivación, el rendimiento, la salud, el bienestar y la seguridad laboral (Grech, Neal, \& Yeo, 2009).

Puede asegurarse que la definición y la medición de la fatiga han sido problemáticas para los investigadores y los clínicos; en este sentido, han proliferado multitud de escalas de medida, en lengua inglesa, de una y de varias dimensiones que posibilitan la evaluación de la población tanto clínica (Dittner et al., 2004) (por ejemplo, enfermos crónicos, psiquiátricos, reumáticos, VIH y cáncer) como laboral (Lerman et al., 2012).

Cabe destacar que uno de los instrumentos desarrollados para evaluar la fatiga es el Multidimensional Fatigue Inventory ([MFI]; Smets, Garssen, Bonke, \& De Haes, 1995; Smets, Garssen, Cull, $\&$ De Haes, 1996). En su origen, se utilizaba en contextos clínicos para la evaluación de la fatiga en pacientes con cáncer. No obstante, también ha sido empleado en el entorno laboral, por ejemplo, para diagnosticar la fatiga en los anestesiólogos (Mansour, Riad, \& Moussa, 2010).

Al hilo de lo anterior, se consideró necesario adaptar un instrumento que hiciese posible evaluar la fatiga dada la escasez de escalas, en español, de este constructo científico. Es necesario tener instrumentos de medida para evaluar, que hagan posible establecer sistemas de vigilancia de la salud en el ámbito del trabajo, con el fin de detectar posibles disfunciones de forma precoz (Dollard, Skinner, Tuckey, \& Bailey, 2007). El MFI puede considerarse una versión breve (Boada-Grau, de Diego, de Llanos-Serra, \& Vigil-Colet, 2011), dado que presenta algunas características como la presentación de una información valiosa, la minimización del sesgo de respuesta relacionado con el cansancio de un instrumento de mayor longitud, la evaluación rápida y sencilla y la reducción del tiempo dedicado a dar respuestas.

Finalmente, el objetivo general ha sido realizar una adaptación al español del original inglés. Los objetivos específicos de este estudio son tres: (1) analizar la estructura interna de la escala, (2) 
comprobar la fiabilidad y (3) aportar evidencias de validez convergente de la escala investigada.

\section{Método}

\section{Participantes}

La muestra multiocupacional está compuesta por 414 empleados de España, de los que el $48.5 \%$ son hombres y el $51.5 \%$, mujeres. Los entrevistados tienen una media de edad de 38.17 años ( $D E=$ 12.18). Su nivel de formación académica se distribuye así: sin estudios y ningún certificado o título académico, el $17.8 \%$; estudios primarios inacabados y certificado de escolaridad, el $41.6 \%$; Bachiller elemental, FP-I o graduado escolar, el 25.7 \%; Bachiller Superior, BUP, FP-II, COU o PAU-Mayores 25 años, el $11.4 \%$; estudios universitarios, el $3.5 \%$. La antigüedad media en el lugar de trabajo actual es de 8.25 años $(D E=9.05)$. La antigüedad media en su profesión es 13.06 años $(D E=11.58)$. Y la antigüedad media en su actual empresa es de 10.44 años $(D E=10.66)$.

\section{Instrumentos}

\section{El Multidimensional Fatigue Inventory ([MFI];} Smets et al., 1995; Smets et al., 1996) en su versión original en inglés tiene 20 ítems y cinco factores. Los factores son: Fatiga General (4 ítems; $\alpha=$ 0.83; por ejemplo, "1.- Me siento en forma"), Fatiga Física (4 ítems; $\alpha=0.85$; por ejemplo, "7.- Tengo un gran aguante físico"), Reducción de la Actividad (4 ítems; $\alpha=0.79$; por ejemplo, "6.- Cuando hago algo, me concentro en ello"), Reducción de la Motivación (4 ítems; $\alpha=0.82$; por ejemplo, "9.Creo que hago muy poco durante el día") y Fatiga Mental (4 ítems; $\alpha=0.91$; por ejemplo, "17.- No me apetece hacer nada"). El formato de respuesta es de 7 puntos que van desde $1=$ Sí, eso es cierto a $7=$ No, eso no es cierto. La validación del instrumento se realizó llevando a cabo los pasos que se señalan en la literatura científica sobre la adaptación de instrumentos de evaluación (Evers et al., 2013; Hambleton, Merenda, \& Spielberger, 2005; Muñiz \& Bartram, 2007): traducción de los ítems al español por expertos, grupo de discusión sobre la traducción realizada de los ítems, retrotraducción de nuevo al inglés (back-translation) y comprobación de la equivalencia entre ambas versiones.

La Fatigue Assessment Scale ([FAS]; Michielsen, De Vries, Van Heck, Van de Vijver, \& Sijtsma, 2004) evalúa la fatiga general. La adaptación española (Boada-Grau, Merino-Tejedor, Gil-Ripoll, \& Vigil-Colet, 2012) indica que es unifactorial y tiene diez ítems ("6.- Me cuesta empezar cosas"), su fiabilidad es de 0.87 , se contesta con una escala de 5 anclajes (de $1=$ Completamente en desacuerdo a 5 = Completamente de acuerdo).

La Fatigue Severity Scale Questionnaire ([FSS]; Krupp, LaRocca, Muir-Nash, \& Steinberg, 1989) diagnostica la fatiga severa. La versión española realizada por Boada-Grau, Merino-Tejedor, GilRipoll y Vigil-Colet (2013) consta de un factor y nueve ítems ("1.- Mi motivación es más baja cuando estoy fatigado/a"), la escala de contestación es de 7 puntos (de $1=$ Completamente en desacuerdo a 7 $=$ Completamente de acuerdo) y el $\alpha$ de Cronbach es de 0.88 .

Además, se incluyeron cinco variables a modo de correlatos externos que permitieron obtener algunas evidencias de validez (Boada-Grau, SánchezGarcía, Prizmic-Kuzmica, \& Vigil-Colet, 2012) de la escala MFI; estos hacen referencia al cansancio, a la falta de energía y a los días que se ha ido a trabajar estando enfermo.

\section{Procedimiento}

Para la escogencia de los participantes, se utilizó el muestreo no probabilístico, también denominado aleatorio-accidental (Kerlinger, 2001). La recogida de datos se realizó desde 2009 hasta 2012. Las escalas se administraron en horario laboral y la participación fue voluntaria. Se les aseguró la confidencialidad y el anonimato de los datos obtenidos.

\section{Análisis de datos}

Para realizar los análisis estadísticos pertinentes, la muestra de 414 empleados fue dividida de forma aleatoria en dos submuestras de 207 sujetos cada 
una. Se realizó un Análisis Factorial Exploratorio (AFE) (submuestra 1) para estudiar la dimensionalidad de la prueba, mediante el método de extracción de análisis de componentes principales y aplicando la rotación Oblimin. Se utilizaron la medida de adecuación muestral de Kaiser-MeyerOlkin $(\mathrm{KMO})$ y la prueba del test de esfericidad de Bartlett.

El Análisis Factorial Confirmatorio (AFC) (submuestra 2) se realizó mediante el Exploratory Structural Equation Modeling ([ESEM ]; Asparouhov \& Muthén, 2009), que es un modelo de ecuaciones estructurales. El modelo ESEM fue elegido ya que es apropiado para analizar medidas típicas y, además, es un modelo semirrestringido que es idóneo para este tipo de datos y proporciona ajustes razonables (Ferrando \& Lorenzo-Seva, 2000; Hopwood \& Donnellan, 2010).

Se utilizó el SPSS 19.0. (Tabla 2) para la realización del AFE (submuestra 1), la evaluación de la consistencia interna de las escalas ( $\alpha$ de Cronbach) y la obtención de evidencias de validez convergente (submuestras 1 y 2). Se aplicó el Mplus (Versión 6.12) para obtener del AFC (ESEM).

\section{Resultados}

El AFE identificó tres factores como solución más apropiada (Tabla 1). Dicha solución mostró buenos índices de adecuación muestral (Kaiser-Meyer-Olkin $=0.901)$ y la prueba de esfericidad de Bartlett $\left(\chi^{2}=1766.752, g l=171, p<0.01\right)$. El scree-test (Cattell, 1966) recomendó una solución de tres factores. A partir de los 20 ítems de la versión original en inglés, se depuró la escala eliminando todos aquelos ítems que presentaran saturaciones inferiores a 0,4 o saturaciones complejas (superiores a 0,4 en más de un factor). En este sentido, se descartó un ítem. La varianza total explicada es del $54.72 \%$.

En la versión española, dada la necesidad de confirmar la estructura del AFE (submuestra 1), se efectuó un AFC (submuestra 2). Los índices son: el error cuadrático medio de aproximación (RM$\mathrm{SEA} \leq 0.06$ ), el índice de ajuste comparado (CFI $\geq 0.95$ ) y el índice de Tucker-Lewis (TLI $\geq 0.95$ ). Los resultados obtenidos presentan un buen ajuste del modelo dado que los índices (RMSEA = 0.06; CFI $=0.95$ y TLI $=0.95$ ) se situan dentro de los valores considerados óptimos. Los modelos de uno, cuatro o más factores han quedado descartados porque no se ajustan.

En la Tabla 2, se detallan la media, la desviación estándar, la fiabilidad, los intervalos de confianza, los correlatos externos y las correlaciones entre los tres factores de la escala MFI y los factores de las escalas FAS y FSS, para las dos submuestras. Las correlaciones del instrumento que se presentaron con las dos escalas anteriores y algunos criterios externos informan de algunas evidencias de validez. Además, con el fin de determinar si existían diferencias significativas entre las correlaciones obtenidas en las dos submuestras se aplicó la transformación z (al 1 \%) de Fisher (Boada-Grau, Prizmic-Kuzmica, Serrano-Fernández, \& Vigil-Colet, 2013); en este sentido, cabe destacar que no se ha apreciado ninguna diferencia significativa entre las mismas.

\section{Discusión}

En el presente estudio, se presentan las propiedades psicométricas de la escala MFI de Smets y sus colaboradores (1995) y Smets et al. (1996), que consta de 19 ítems, es un instrumento que permite evaluar la fatiga a través de tres factores (Fatiga General, Fatiga Física y Concentración/Motivación). En este sentido, el objetivo general se ha cumplido dado que se ha realizado la adaptación de dicha escala al español en una muestra heterogénea de empleados.

El primer objetivo específico se ha constatado, ya que se ha analizado la estructura interna (AFE y replicación con AFC) configurándose tres factores. El primer factor, denominado Fatiga General ( $\alpha$ de Cronbach $=0.7$ y 0.71 según submuestra 1 y 2) está relacionado con aquellos aspectos que hacen referencia a la inapetencia, la falta de concentración, bajo rendimiento y el cansancio mental. Es el que tiene más varianza explicando el $39.22 \%$ y está integrado por ocho ítems (números: 2, 8, 9, 12, $15,16,17$ y 18). Al segundo factor, llamado Fatiga Física hace alusión a los aspectos físicos como la condición, la forma, la tenacidad y la actividad. Su 
TABLA 1

Escala MFI: Matriz de saturaciones de los factores extraídos del análisis factorial exploratorio

\begin{tabular}{|c|c|c|c|c|}
\hline Ítems & Original Inglés & $F 1^{\mathrm{a}}$ & $\mathrm{F} 2^{\mathrm{b}}$ & F3c \\
\hline 1.-Me siento en forma [I feel fit] & F1.Fatiga General & -0.13 & 0.63 & 0.16 \\
\hline $\begin{array}{l}\text { 2.-Físicamente, me siento capaz de poco [Physically I feel only able to do } \\
\text { a little] }{ }^{\mathrm{d}}\end{array}$ & F2.Fatiga Física & 0.47 & -0.3 & 0.04 \\
\hline 3.-Me siento muy activo [I feel very active] & F4.Reducción Actividad & -0.25 & 0.4 & 0.3 \\
\hline $\begin{array}{l}\text { 4.-Me apetece hacer todo tipo de cosas agradables [I feel like doing all } \\
\text { sorts of nice things] }\end{array}$ & F5.Reducción Motivación & -0.13 & 0.21 & 0.56 \\
\hline $\begin{array}{l}\text { 5.-Creo que hago muchas cosas a lo largo del día [I think I do a lot in a } \\
\text { day] }\end{array}$ & F4.Reducción Actividad & 0.04 & -0.1 & 0.78 \\
\hline $\begin{array}{l}\text { 6.-Cuando hago algo, me concentro en ello [When I am doing } \\
\text { something, I can keep my thoughts on it] }\end{array}$ & F3.Fatiga Mental & 0.01 & 0.01 & 0.84 \\
\hline 7.-Tengo un gran aguante físico [Physically I can take on a lot]. & F2.Fatiga Física & -0.09 & 0.56 & 0.23 \\
\hline $\begin{array}{l}\text { 8.-No quiero ni pensar en tener que hacer cosas [I dread having to do } \\
\text { things] }\end{array}$ & F5.Reducción Motivación & 0.70 & 0.02 & -0.13 \\
\hline $\begin{array}{l}\text { 9.-Creo que hago muy poco durante el día [I think I do very little in a } \\
\text { day }{ }^{\mathrm{d}}\end{array}$ & F4.Reducción Actividad & 0.71 & 0.15 & -0.07 \\
\hline 10.-Me puedo concentrar bien [I can concentrate well]. & F3.Fatiga Mental & -0.05 & 0.12 & 0.56 \\
\hline 11.-Estoy descansado/a [I am rested]. & F1.Fatiga General & 0.12 & 0.57 & 0.06 \\
\hline $\begin{array}{l}\text { 12.-Me cuesta mucho concentrarme en las cosas [It takes a lot of effort } \\
\text { to concentrate on things] }{ }^{\mathrm{d}}\end{array}$ & F3.Fatiga Mental & 0.72 & 0.01 & 0.02 \\
\hline 13.-Estoy en baja forma física [Physically I feel I am in a bad condition] ${ }^{\mathrm{d}}$ & F2.Fatiga Física & 0.33 & -0.64 & 0.09 \\
\hline 14.-Tengo un montón de planes [I have a lot of plans]. & F5.Reducción Motivación & -0.15 & 0.15 & 0.4 \\
\hline 15.-Me canso con facilidad [I tire easily $]^{\text {d }}$ & F1.Fatiga General & 0.6 & -0.35 & 0.06 \\
\hline 16.-Rindo poco [I get little done $]^{\mathrm{d}}$ & F4.Reducción Actividad & 0.75 & 0.16 & -0.18 \\
\hline 17.-No me apetece hacer nada[I don't feel like doing anything] ${ }^{\mathrm{d}}$ & F5.Reducción Motivación & 0.85 & -0.04 & 0 \\
\hline $\begin{array}{l}\text { 18.-Se me van los pensamientos con facilidad [My thoughts easily } \\
\text { wander] }^{\mathrm{d}}\end{array}$ & F3.Fatiga Mental & 0.61 & -0.09 & 0.03 \\
\hline $\begin{array}{l}\text { 19.-Me encuentro en una excelente condición física [Physically I feel I } \\
\text { am in an excellent condition] }\end{array}$ & F2.Fatiga Física & 0.07 & 0.88 & 0.06 \\
\hline Me siento cansado/a [Versión inglesa número 5.-I feel tired] d,e & F1.Fatiga General & & & \\
\hline
\end{tabular}

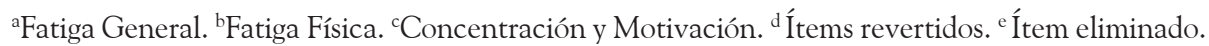

Fuente: elaboración propia

fiabilidad es de 0.82 y 0.8 (según submuestra 1 y 2). Está configurado por cinco ítems (números: 1 , $3,7,13$ y 19) que explican el $8.48 \%$ de la varianza. Y el último factor, Concentración/Motivación hace referencia a las ganas y a la apetencia de hacer cosas, a la iniciativa y a los planes de futuro. Tiene cinco ítems (números: 4, 5, 6, 10 y 14) y su fiabilidad es de 0.74 y 0.71 (según submuestra). La varianza explicada es del $7.01 \%$. Las correlaciones entre los tres factores en las dos submuestras oscilan desde -0.44 hasta 0.39. Cabe destacar que los resultados del AFE y del AFC no validan el modelo original inglés (Smets et al., 1995; Smets et al., 1996) de cinco factores ni la adaptación al francés de cuatro factores (Gentile, Delarozière, Favre, Sambuc, \& San Marco, 2003).

El segundo objetivo específico se verifica ya que los coeficientes de fiabilidad obtenidos (en las dos submuestras) de nuestra investigación oscilan entre 0.7 y 0.82. Las $\alpha$ Cronbach (Cronbach, 1951) se consideran aceptables si son iguales o superiores a 0.7. En la versión original inglesa (Smets et al., 1995; Smets et al., 1996), en distintas muestras (por ejemplo, pacientes de radioterapia, pacientes con fatiga crónica, estudiantes de psicología y medicina y soldados de la armada), la fiabilidad oscila entre 0.53 y 0.93 . Y en la versión francesa (Gentile et al., 2003), el $\alpha$ de Cronbach se encuentran entre 0.68 y 0.92 . 


\section{TABLA 2}

Factores de la escala MFI-19: estadísticos descriptivos, fiabilidad, intervalos de confianza y correlaciones con las variables sociodemográficas y los factores de las escalas (FAS y

FSS) y matriz de correlaciones entre los tres factores de la escala analizada

\begin{tabular}{|c|c|c|c|c|c|c|}
\hline & \multicolumn{3}{|c|}{ Submuestra $1(N=207)$} & \multicolumn{3}{|c|}{ Submuestra $2(N=207)$} \\
\hline & $\mathrm{F} 1^{\mathrm{a}}$ & $\mathrm{F} 2^{\mathrm{b}}$ & F3c & $\mathrm{F} 1$ & F2 & F3 \\
\hline Media & 22.32 & 18.96 & 12.86 & 22.2 & 18.97 & 12.27 \\
\hline DE & 7.2 & 7.77 & 6.09 & 6.76 & 7.8 & 6.12 \\
\hline Fiabilidad & 0.7 & 0.82 & 0.74 & 0.71 & 0.8 & 0.71 \\
\hline Intervalo de Confianza & $0.68-0.73$ & $0.78-0.85$ & $0.69-0.79$ & $0.69-0.74$ & $0.75-0.84$ & $0.66-0.77$ \\
\hline $\begin{array}{l}\text { ¿Cuántos días te has sentido cansado o sin energía } \\
\text { durante la semana pasada? }\end{array}$ & $0.27 * *$ & $0.21 * *$ & 0.05 & $0.24 * *$ & $0.19 * *$ & 0.07 \\
\hline $\begin{array}{l}\text { ¿Cuántas veces te has sentido cansado/a o sin } \\
\text { energía durante más de } 3 \text { horas en cualquier día } \\
\text { de la semana pasada? }\end{array}$ & $0.31 * *$ & $0.26 * *$ & 0.12 & $0.29 * *$ & $0.24 * *$ & 0.11 \\
\hline $\begin{array}{l}\text { ¿Cuántas veces, te has sentido tan cansado/a hasta } \\
\text { el punto qué has tenido que obligarte a ti mismo/a } \\
\text { para hacer cosas durante la semana pasada? }\end{array}$ & $0.17 * *$ & $0.14 *$ & 0.1 & $0.19 * *$ & $0.15^{*}$ & 0.05 \\
\hline $\begin{array}{l}\text { ¿Cuántas veces, te has sentido/a cansado/a o sin } \\
\text { energía al hacer cosas con las que disfrutas duran- } \\
\text { te la última semana? }\end{array}$ & $0.27 * *$ & $0.23 * *$ & 0.07 & $0.26 * *$ & $0.22 * *$ & 0.07 \\
\hline ¿Cuantos días has ido al trabajo estando enfermo? & $0.22 * *$ & 0.06 & 0.11 & $0.21 * *$ & 0.09 & 0.06 \\
\hline FAS & $0.57 * *$ & $0.53 * *$ & $0.34 * *$ & $0.49 * *$ & $0.47 * *$ & $0.31 * *$ \\
\hline FSS & $0.4 * *$ & $0.37 * *$ & $0.21 * *$ & $0.31 * *$ & $0.25 * *$ & 0.05 \\
\hline F1 & - & - & - & - & - & - \\
\hline F2 & $-0.39 *$ & - & - & $-0.35^{*}$ & - & \\
\hline F3 & $-0.44 *$ & $0.35 *$ & - & $-0.4^{*}$ & $0.39 *$ & - \\
\hline
\end{tabular}

$* * p<0.01 * p<0.05$.

aFatiga General

bFatiga Física

cConcentración y Motivación

Fuente: elaboración propia

También se confirma el tercer objetivo específico, ya que se encuentran evidencias de validez convergente. En términos generales, los datos resultantes del estudio (en las dos submuestras) indican que los tres factores de la escala adaptada se asocian de forma significativa a otras variables. Se obtiene un total de 29 correlaciones, todas ellas directas, entre los tres factores de la escala con otros correlatos externos (por ejemplo, "días que se ha sentido cansado o sin energía", "obligarse a sí mismo e ir al trabajo estando enfermo") y con otras dos escalas de contraste específicas fatiga (FAS y FSS). Las correlaciones significativas oscilan entre $r=0.14$ $(p<0.05)$ y $r=0.57(p<0.01)$.

En conclusión, teniendo en cuenta los resultados obtenidos en la presente investigación, se puede concluir que la escala MFI, en la versión española, presenta unas aceptables propiedades psicométricas y permite evaluar la fatiga en el entorno laboral. La presente escala se conforma como un instrumento breve, de rápida aplicación y de fácil interpretación a través de las tres subescalas (Fatiga General, Fatiga Física y Concentración/Motivación) que la componen; es más, posibilita una valoración independiente de cada una de ellas. Por un lado, la versión actual del MFI se configura como trifactorial y reduce el número de ítems, pasando de los 20 del original inglés a 19. Además, los coeficientes de fiabilidad de los tres factores son adecuados. Y, por último, se han hallado evidencias de validez con otros correlatos y escalas de comparación. 
En cuanto a la aplicación de la presente escala de evaluación en el entorno laboral puede tener varios vectores. En primer lugar, la escala MFI puede ser tenida en cuenta para evaluar la vigilancia de la salud basada en la evidencia (Boada-Grau, de Diego, \& Agulló, 2004; Verbeek et al., 2002), así facilita la toma de decisiones en la prevención de la salud, fundamentada en pruebas científicas contrastadas que cumplan requisitos psicométricos óptimos (Boada-Grau, González, Vigil-Colet, Mañas, \& Agulló, 2009; Boada-Grau, Merino-Tejedor et al., 2012; Spencer, Robertson, \& Folkard, 2006), todo ello con el propósito de proteger la salud de los empleados y de prevenir los posibles riesgos laborales.

Por otro lado, la Organización Internacional del Trabajo (OIT, 2001) considera que las evaluaciones, entre ellos los riesgos psicosociales, deben transitar desde lo cualitativo, tan mayoritario en este ámbito, hacia lo cuantitativo, y por lo expuesto en el estudio la presente escala contribuye a ello.

Tercero, ya que la escala presenta unas propiedades psicométricas óptimas, puede permitir a los profesionales de la prevención adquirir datos destinados a diseñar e implementar programas preventivos (Machin \& Hoare, 2008) y de intervención (OIT, 2001).

Y finalmente, la escala MFI posibilita la realización de investigaciones con una metodología multinivel que debería escudriñar de qué forma variables como la cultura organizacional, el estilo de liderazgo de los mánagers y el clima de los equipos de trabajo pueden ser predictoras de la fatiga laboral. Además, hace posible estudios longitudinales que permitan descubrir los efectos sobre la salud, y de forma singular sobre la fatiga laboral, de las regulaciones de empleo (downsizing), la inseguridad en el trabajo y la temporalidad (García, 2010).

La presente investigación tiene algunas limitaciones. La primera de ellas es la utilización del autoinforme que puede haber provocado el incremento de la asociación entre las variables debido a la varianza del método común (Podsakoff, MacKenzie, Lee, \& Podsakoff, 2003); no obstante, se han incorporado correlatos externos que de forma mayoritaria correlacionan con la escala MFI. El uso del autoinforme en las investigaciones sobre la fatiga ha sido ampliamente utilizado (Gentile et al., 2003; Smets et al., 1995; Smets et al., 1996). La utilización de este permite poder captar con precisión la percepción y las actitudes individuales sobre el constructo investigado. En próximas investigaciones, deberían de incluirse las evaluaciones que los compañeros de trabajo y/o supervisores pueden realizar sobre un empleado.

La segunda limitación hace referencia a que sería preciso analizar la validez discriminante atendiendo tanto al tipo de trabajo ("cuello azul" vs "cuello blanco") como en diferentes colectivos de trabajadores inmigrantes de origen cultural diverso (por ejemplo, africano, latinoamericano y centroeuropeo).

La tercera limitación se focalizaría en el procedimiento de muestreo. En este sentido, sería preciso utilizar procedimientos de muestreo aleatorio, con el fin de poder aumentar la validez externa. Sin embargo, la utilización de muestras no probabilísticas no presenta una amenaza importante para la validez del estudio (Highhouse \& Gillespie, 2008).

Y por último, sería preciso analizar si algunos aspectos de los empleados como, la impulsividad funcional y/o disfuncional, el atrevimiento, la agresividad, el burnout, las manifestaciones psicosomáticas y el neuroticismo podrían relacionarse con la fatiga laboral.

\section{Referencias}

Asparouhov, T., \& Muthén, B. (2009). Exploratory structural equation modeling. Structural Equation Modeling, 16(3), 397-438.

Beurskens, A. J., Bültmann, U., Kant, I. J., Vercoulen, J. H., Bleijenberg, G., \& Swaen, G. M. (2000). Fatigue among working people: Validity of a questionnaire measure. Occupational and Environmental Medicine, 57(5), 353-357.

Boada-Grau, J., de Diego, R., \& Agulló, E. (2004). Burnout and psychosomatics manifestations as consequences of organizational climate and labour motivation. Psicothema, 16(1), 125-131.

Boada-Grau, J., de Diego, R., de Llanos-Serra, E., \& Vigil-Colet, A. (2011). Versión breve en español del Team Climate Inventory (TCI-14): desarrollo 
y propiedades psicométricas. Psicothema, 23(2), 308-313.

Boada-Grau, J., González, S., Vigil-Colet, A., Mañas, M. A., \& Agulló, S. (2009). CONS-32: Estructura factorial y propiedades psicométricas. Psicothema, 21(1), 165-169.

Boada-Grau, J., Merino-Tejedor, E., Gil-Ripoll, C., \& Vigil-Colet, A. (2012). Adaptación al español de la Escala de Evaluación de la Fatiga. Manuscrito no publicado. Universitat Rovira i Virgili, Tarragona, España.

Boada-Grau, J., Merino-Tejedor, E., Gil-Ripoll, C., \& Vigil-Colet, A. (2013). Adaptación al español del Cuestionario de Fatiga Severa. Manuscrito no publicado. Universitat Rovira i Virgili, Tarragona, España.

Boada-Grau, J., Prizmic-Kuzmica, A.J., Serrano-Fernández, M.J. y Vigil-Colet, A. (2013). Factor structure, reliability and validity of the workaholism battery (WorkBAT): Spanish version. Anales de Psicología, 29, 923-933.

Boada-Grau, J., Sánchez-García, J. C., Prizmic-Kuzmica, A. J., \& Vigil-Colet, A. (2012). Work health and hygiene in the transport industry (TRANS-18): Factorial structure, reliability and validity. The Spanish Journal of Psychology, 15(1), 357-366.

Cattell, R. B. (1966). The scree test for the number of factors. Multivariate Behavioral Research, 1(2), 245-276.

Cronbach, L. J. (1951). Coefficient alpha and the internal structure of tests. Psychometrika, 16(3), 297-332.

Dittner, A. J., Wessely, S. C., \& Brown, R. G. (2004). The assessment of fatigue: A practical guide for clinicians and researchers. Journal of Psychosomatic Research, 56(2), 157-170.

Dollard, M., Skinner, N., Tuckey, M. R., \& Bailey, T. (2007). National surveillance of psychosocial risk factors in the workplace: An international overview. Work Ë Stress, 21(1), 1-29.

Evers, A., Muñiz, J., Hagemeister, C., Høstmælingen, A., Lindley, P., Sjöberg, A., \& Bartram, D. (2013). Assessing the quality of tests: Revision of the EFPA review model. Psicothema, 25(3), 283-291.

Ferrando, P. J., \& Lorenzo-Seva, U. (2000). Unrestricted versus restricted factor analysis of multidimensio- nal test items: some aspects of the problem and some suggestions. Psicológica, 21(2), 301-323.

García, A. (2010). Mercado laboral y salud (Informe SESPAS 2010). Gaceta Sanitaria, 24(Supl. 1), 62-67.

Gentile, S., Delarozière, J. C., Favre, F., Sambuc, R., \& San Marco, J. L. (2003). Validation of the French 'multidimensional fatigue inventory' (MFI 20). European Journal of Cancer Care, 12(1), 58-64.

González, J. L., Moreno, B., Garrosa, E., \& López, A. (2005). Spanish version of the Swedish Occupational Fatigue Inventory (SOFI): Factorial replication, reliability and validity. International Journal of Industrial Ergonomics, 35(8), 737-746.

Grech, M. R., Neal, A., \& Yeo, G. (2009). An examination of the relationship between workload and fatigue within and across consecutive days of work: Is the relationship static or dynamic? Journal of Occupational Health Psychology, 14(3), 231-242.

Hambleton, R. K., Merenda, P. F., \& Spielberger, C. D. (2005). Adapting educational and psychological tests for cross-cultural assessment. London: Erlbaum.

Herusasongko, B., Sutomo, A. H., \& Sudibyakto, H. (2012). Effects of the occupational physical environmental conditions and the individual characteristics of the workers on occupational stress and fatigue. International Journal of Public Health Science, 1(2), 61-68.

Highhouse, S., \& Gillespie, J. Z. (2008). Do samples really matter that much? En C. E. Lance \& R. J. Vandenberg (Eds.), Statistical and methodological myths and urban legends: Received doctrine, verity, and fable in the organizational and social sciences (pp. 247-266). Mahwah, NJ: Erlbaum.

Hopwood, C. J., \& Donnellan, M. B. (2010). How should the internal structure of personality inventories be evaluated? Personality and Social Psychology Review, 14(3), 332-346.

Kerlinger, F. N. (2001). Investigación del comportamiento: métodos de investigación en ciencias sociales. México: McGraw-Hill.

Krupp, L. B., LaRocca, N. G., Muir-Nash, J., \& Steinberg, A.D. (1989). The Fatigue Severity Scale. Application to patients with multiple sclerosis and systemic lupus erythematosus. Archives of Neuro$\log y, 46(10), 1121-1123$. 
Lerman, S. E., Eskin, E., Flower, D. J., George, E. C., Gerson, B., Hartenbaum, N.,... Moore-Ede, M. (2012). Fatigue Risk Management in the workplace. Journal of Occupational and Environmental Medicine, 54(2), 231-258.

Machin, M. A., \& Hoare, P. N. (2008). The role of workload and driver coping styles in predicting bus drivers' need for recovery, positive and negative affect, and physical symptoms. Anxiety, Stress and Coping, 21(4), 359-375.

Mansour, A., Riad, W., \& Moussa, A. (2010). The occupational fatigue in anesthesiologists: Illusion or real? Middle East Journal of Anesthesiology, 20(4), 530-534.

Michielsen, H. J., De Vries, J., Van Heck, G. L., Van de Vijver, F. J. R., \& Sijtsma, K. (2004). Examination of the dimensionality of fatigue. The construction of the Fatigue Assessment Scale (FAS). European Journal of Psychological Assessment, 20(1), 39-48.

Muñiz, J., \& Bartram, D. (2007). Improving international tests and testing. European Psychologist, 12(3), 206-219.

Organización Internacional del Trabajo. (2001). Proyecto de directrices técnicas de la OIT sobre sistemas de gestión de la seguridad y salud en el trabajo. Ginebra: Autor.

Podsakoff, P. M., MacKenzie, S. B., Lee, J. -Y., \& Podsakoff, N. P. (2003). Common method biases in behavioral research: A critical review of the literature and recommended remedies. Journal of Applied Psychology, 88(5), 879-903.
Roach, G. D., Fletcher, A., \& Dawson, D. (2004). A model to predict work-related fatigue based on hours of work. Aviation, Space, and Environmental Medicine, 75(3), 61-69.

Satterfield, B. C., \& Van Dongen, H. P. A. (2013). Occupational fatigue, underlying sleep and circadian mechanisms, and approaches to fatigue risk management fatigue. Biomedicine, Health 83 Behavior, 1(3), 118-136.

Smets, E. M. A., Garssen, B., Bonke, B., \& De Haes, J. C. (1995). The Multidimensional Fatigue Inventory (MFI): Psychometric qualities of an instrument to assess fatigue. Journal of Psychosomatic Research, 39(3), 315-325. http://dx.doi.org/10.1016/0022. 3999(94)00125-O

Smets, E. M. A., Garssen, B., Cull, A., \& De Haes, J. C. (1996). Application of the multidimensional fatigue inventory (MFI 20) in cancer patients receiving radiotherapy. British Journal of Cancer, 73(2), 241-245.

Spencer, M. B., Robertson, K. A., \& Folkard, S. (2006). The development of a fatigue/risk index for shiftworkers (Research Rep. 446). Sudbury, England: HSE Books.

Verbeek, J. H., Van Dijk, F. J., Mailmivaara, A., Hulshof, C. T., Räsänen, K., Kankaanpää, E. E., \& Mukala, K. (2002). Evidence-based medicine for occupational health. Scandinavian Journal of Work Environment Health, 28(3), 197-204. 
